\title{
Nutrition et déficit énergétique
}

\section{Nutrition and energy deficit}

(C) SRLF et Springer-Verlag France 2013

\section{FC041}

\section{Intérêt de l'échographie gastrique dans l'évaluation} du résidu gastrique des patients de réanimation M. Danguy des Déserts ${ }^{1}$, S. Huynh-Moynot ${ }^{1}$, E. Montelescaut ${ }^{1}$, G. Prat ${ }^{2}$, A. Renault ${ }^{2}$, M. Ould-Ahmed ${ }^{1}$, B.V. Nguyen ${ }^{1}$ ${ }^{1}$ Fédération anesthésie-réanimation urgences, hôpital d'instruction des armées Clermont-Tonnerre, Brest, France

${ }^{2}$ Réanimation médicale, CHU de la Cavale-Blanche, Brest, France

Introduction : La nutrition entérale (NE) est un élément important de la prise en charge des patients en réanimation. La surveillance de la tolérance de la NE s'effectue habituellement par mesure du volume résiduel gastrique (VRG) par aspiration à la seringue via la sonde nasogastrique (SNG). Cette technique imparfaite pourrait être remplacée par la mesure échographique de la surface antrale (SA). L'objectif de notre étude était d'évaluer la corrélation entre la mesure échographique de la SA et la mesure du VRG par aspiration à la seringue.

Patients et méthodes : Les patients hospitalisés en réanimation ayant bénéficié d'une NE via une SNG ont été inclus dans cette étude prospective, bicentrique. Les critères d'exclusion étaient l'âge $<18$ ans, un antécédent de gastrectomie ou d'œsophagectomie. Une mesure quantitative de la lumière interne de l'antre gastrique notée SA était réalisée à l'aide d'un échographe Phillips ${ }^{\circledR} \mathrm{CX} 50$ ou iE33 et d'une sonde type abdominale C5-1. La facilité de réalisation de la mesure (facile, difficile ou impossible) était notée par l'opérateur réalisant l'échographie. Puis une aspiration du contenu gastrique par la SNG avec une seringue de $50 \mathrm{~mL}$ était effectuée par l'infirmier(e), le volume aspiré était noté VRG. Les couples de mesures (VRG et SA) étaient ensuite comparés.

Résultats : 64 patients ont été inclus. L'âge moyen était de $65,3 \pm 14,1$ ans, l'index de gravité simplifié II moyen de 57,1 $\pm 16,7$, le poids moyen de 76,2 $\pm 19,2 \mathrm{kgs}$, l'index de masse corporelle moyen de $26,7 \pm 6,2 \mathrm{kgs} / \mathrm{m}^{2} .96$ couples de mesures VRG/SA ont été

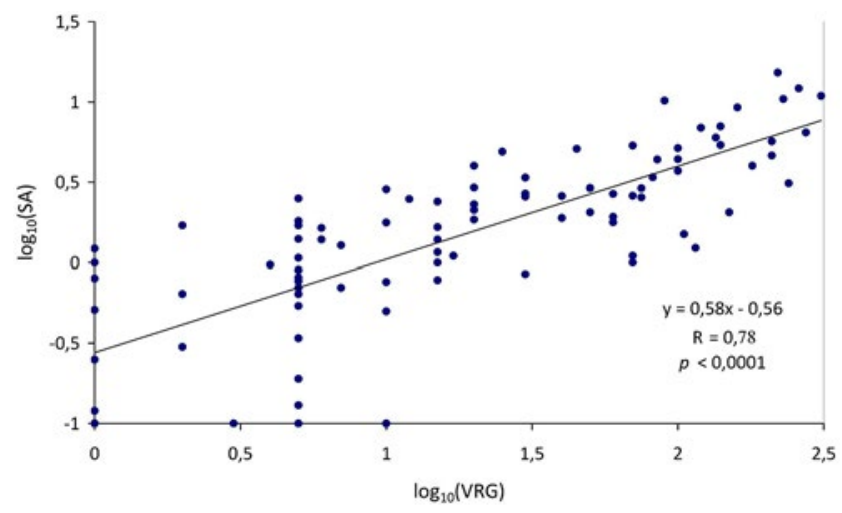

réalisés. Le VRG moyen était de 53,9 $\pm 72,5 \mathrm{~mL}$, allant de 0 à $310 \mathrm{~mL}$. La mesure moyenne de SA était de $2,7 \pm 2,9 \mathrm{~cm}^{2}$, allant de 0 à $15,26 \mathrm{~cm}^{2}$. Il existe une corrélation statistiquement significative entre $\log _{10}(\mathrm{VRG})$ et $\log _{10}(\mathrm{SA})(\mathrm{R}=0,78 ; p<0,0001)$.

La mesure échographique a été possible chez $96 \%$ des patients et jugée facile dans $79 \%$ des cas.

Conclusion : Notre étude suggère que la mesure échographique de la SA serait un reflet du VRG mesuré par aspiration à la seringue et pourrait faire partie des examens échographiques utilisés en réanimation. Cette technique permettrait d'éviter les interruptions d'administration de la NE liées aux aspirations nécessaires à la mesure du VRG et pourrait être enseignée aux infirmiers de réanimation.

\section{Bibliographie}

1. Martindale RG, McClave SA, Vanek VW, et al (2009) Guidelines for the provision and assessment of nutrition support therapy in the adult critically ill patient: Society of Critical Care Medicine and American Society for Parenteral and Enteral Nutrition: Executive Summary. Crit Care Med 37:1757-61

2. Perlas A, Chan VW, Lupu CM, et al (2009) Ultrasound assessment of gastric content and volume. Anesthesiology 111:82-9

\section{FC042}

Absence de mesure du résidu gastrique et risque de pneumopathie nosocomiale chez les patients en état de choc traités par amine vasoactive, ventilation mécanique et nutrition entérale

J. Reignier ${ }^{1}$, A. Le Gouge ${ }^{2}$, J.-P. Frat ${ }^{3}$, T. Boulain ${ }^{4}$, E. Mercier ${ }^{5}$, A. Desachy ${ }^{6}$, G. Plantefève ${ }^{7}$, J.-P. Quenot ${ }^{8}$, F. Bellec ${ }^{9}$, M. Clavel $^{10}$, J.-B. Lascarrou ${ }^{11}$

${ }^{1}$ Réanimation, CHD de la Vendée, La Roche-sur-Yon, France ${ }^{2}$ Cic Inserm 202, CHU de Tours, hôpital Bretonneau, Tours, France

${ }^{3}$ Service de réanimation médicale, CHU de Poitiers, Poitiers, France

${ }^{4}$ Service de réanimation polyvalente, CHR, hôpital de la Source, Orléans, France

${ }^{5}$ Service de réanimation médicale, $\mathrm{CHU}$ de Tours, hôpital Bretonneau, Tours, France

${ }^{6}$ Service de réanimation polyvalente, $\mathrm{CH}$ d'Angoulême, Angoulême, France

${ }^{7}$ Service de réanimation polyvalente, $\mathrm{CH}$ d'Argenteuil, Argenteuil, France

${ }^{8}$ Service de réanimation médicale, $\mathrm{CHU}$ de Dijon, complexe du Bocage, Dijon, France

${ }^{9}$ Réanimation polyvalente, $\mathrm{CHG}$, Montauban, France

${ }^{10}$ Réanimation, CHU Dupuytren, Limoges, France

${ }^{11}$ Service de réanimation polyvalente, CHD les Oudairies, La Roche-sur-Yon, France 
Introduction : L'étude NUTRIREA1 a montré que l'absence de surveillance du volume gastrique résiduel (VGR) chez les patients traités par ventilation mécanique (VM) et nourris précocement par voie entérale n'est pas associée à un risque accru de pneumopathies acquises sous ventilation mécanique (PAVM) malgré une fréquence accrue de vomissements chez les patients dont le VGR n'est pas surveillé. Néanmoins, un doute subsiste chez les patients les plus graves, en particulier ceux en état de choc, particulièrement à risque d'intolérance digestive et de PAVM. L'objectif de ce travail est donc de vérifier l'hypothèse que l'absence de surveillance du VGR n'augmente pas le risque de PAVM chez les patients ventilés, traités par amine vasoactive pour un état de choc et nourris précocement par voie entérale.

Patients et méthodes : Il s'agit d'une étude ancillaire de l'essai randomisé, multicentrique NUTRIREA1. Inclusion : tous les patients devant être nourris par sonde nasogastrique dans les 36 heures suivant leur intubation, pour une durée prévisible d'au moins 48 heures et traités par amine vasoactive pendant au moins 2 jours pendant la période de $\mathrm{J} 0$ à $\mathrm{J} 3$. Exclusions : chirurgie digestive récente $(<1$ mois), antécédents de gastrectomie, œsophagectomie ou duodéno-pancréatectomie, présence de gastrostomie ou jéjunostomie, hémorragie digestive active. La NE est débutée le plus tôt possible après l'intubation avec un objectif calorique de $20-25 \mathrm{kcal} / \mathrm{kg} / \mathrm{j}$, pendant la phase aigue puis $25-30 \mathrm{ml} / \mathrm{kg} / \mathrm{j}$. Elle est administrée à débit continu, sur 24 heures, par une sonde nasogastrique siliconée de calibre 14 French. Les modalités de détection de l'intolérance de la NE sont attribuées par tirage au sort. Groupe VGR+ : surveillance des régurgitations ou vomissements et mesure régulière du résidu gastrique (limite : $250 \mathrm{ml} / 6$ heures) ; groupe VGR- : surveillance des régurgitations ou vomissements uniquement. Le critère de jugement principal est le taux de patients ayant présenté au moins une PAVM.

Résultats : Sur les 452 patients inclus dans NUTRIREA1, 259 patients ont été traités par amine vasoactive pendant au moins 2 jours pendant la période de $\mathrm{J} 0$ à $\mathrm{J} 3$ et ont été inclus dans la présente étude. 130 patients VGR+ et 129 VGR- ont été analysés en intention de traiter. Les caractéristiques à l'inclusion des patients VGR+ et VGR- étaient comparables (Age : $64 \pm 13$ et $63 \pm 14$; sexe masculin : $70 \%$ et $68,2 \%$; IMC : $28,1 \pm 8,7$ et $27,0 \pm 6,5$; antécédent de diabète : $23,0 \%$ et $19,3 \%$; IGSII : $53 \pm 17$ et $52 \pm 17$; SOFA : $10 \pm 3$ et $9 \pm 3$; sédation : $91,5 \%$ et $85,2 \%$; curarisation : $29,2 \%$ et $31,7 \%$; insulinothérapie : $62,3 \%$ et $60,4 \%$; dialyse : $6,1 \%$ et $5,4 \%$; lactates : $2,6 \pm 2,3$ et $2,7 \pm 2,1 ;$ Fi02 : $59 \pm 23$ et $60 \pm 23$ ). Les taux de patients ayant eu au moins une PAVM ne différaient pas significativement entre les deux groupes (VGR+ : 17,0 \% et VGR-: $23,3 \% ; p=0,21)$. De même, il n'était pas observé de différence significative pour les densités d'incidence de PAVM (VGR+ : 12,5 et VGR- : 19,9 PAVM / 1000 jours de VM) et le nombre de PAVM observées dans chaque groupe (VGR+ : 23 PAVM et VGR- : 26 PAVM ; RR : 1,14, p=0,69). Le taux des patients ayant présenté au moins un vomissement était par contre plus élevé dans le groupe VGR- $(46,5 \%)$ que dans le groupe VGR+ $(25,4 \%)$. Le nombre de jours avec vomissement et le nombre total d'épisodes de vomissements étaient aussi plus élevés dans le groupe VGR- que dans le groupe VGR+.

Conclusion : Ce travail conforte 1'hypothèse que la surveillance du VGR n'est pas un moyen efficace pour réduire le risque de PAVM chez les patients choqués, ventilés et nourris précocement par voie entérale. Et ceci même si la surveillance du VGR est associée à une réduction de la fréquence des vomissements. Néanmoins, une étude avec un effectif de patients adapté serait nécessaire pour affirmer complètement l'équivalence entre mesure et absence de mesure du VGR quant au risque de PAVM.

\section{FC043}

\section{Resting energy expenditure is correlated with blood C-reactive protein in sepsis}

C. Hickmann, J.Roeseler, D. Castanares-Zapatero, P.F. Laterre Service de soins intensifs, cliniques universitaires Saint-Luc, Bruxelles, Belgique

Introduction: Measured resting energy expenditure by indirect calorimetry has been shown to be considerably increased in critically ill when compared with predictive equations. Correction factors proposed for several critical conditions seem not to be adequate since do not take into account changes occurring during illness evolution. Resting energy expenditure determination is of high relevance to avoid both overfeeding and underfeeding. We conducted an observational study to assess energy cost associated with early exercise. From this study resting energy expenditure was extracted and correlated with routine biomarkers.

Patients and methods: This study was performed in a medicalsurgical Intensive Care Unit in hemodynamically stable and in healthy volunteers. Resting energy expenditure determined by indirect calorimetry (Deltatrac II) was compared with three predictive formulas and correlated in patients with $\mathrm{C}$-reactive protein, creatinine and lactate.

Results: Sixty patients were enrolled but only forty-nine patients with stable and satisfactory indirect calorimetry determination were kept for final analyzes. Forty-one patients were male, mean age was $60 \pm 17$; mean APACHE score was $19 \pm 7$, sixteen receive sedative medication and seventeen vasopressors, nineteen patients were mechanically ventilated and all of them with pressure support mode. Fifteen healthy volunteers were included as a control group. Resting energy expenditure determined by indirect calorimetry was higher than predicted by Harris-Benedict $(18 \pm 18 \%, p<0.001)$ and Fleisch equations $(14 \pm 19 \%, \mathrm{p}<0.001)$ in all patients and lower than Faisy-Fagon equation $(16 \pm 9 \%, p<0.05)$ in the nineteen ventilated patients. In healthy volunteers, no difference was found between measured and estimated values. A correlation was found between measured resting energy expenditure and C-reactive protein $(r=0.41, p=0.004)$. In thirty-one patients with sepsis at the time of assessment, this correlation was more pronounced $(\mathrm{r}=0.47, \mathrm{p}=0.008)$. Difference between Harris-Benedict predictions and indirect calorimetry determination were also correlated with this inflammatory marker in all patients $(\mathrm{r}=0.44, \mathrm{p}=0.001)$, in patients with sepsis $(\mathrm{r}=0.54, \mathrm{p}=0.002)$ and when values from patients with sepsis were standardized for body weight $(r=0.51, p=0.003$ ) (figure). In patients without sepsis, no correlation was found. No correlation between resting energy expenditure and the other blood biomarkers was observed.

Discussion: These results confirm that critically ill often display increased resting energy expenditure compared with predicted values. On the other hand, an equation created for mechanically ventilated critically ill seems to overestimate nutritional needs. This could be

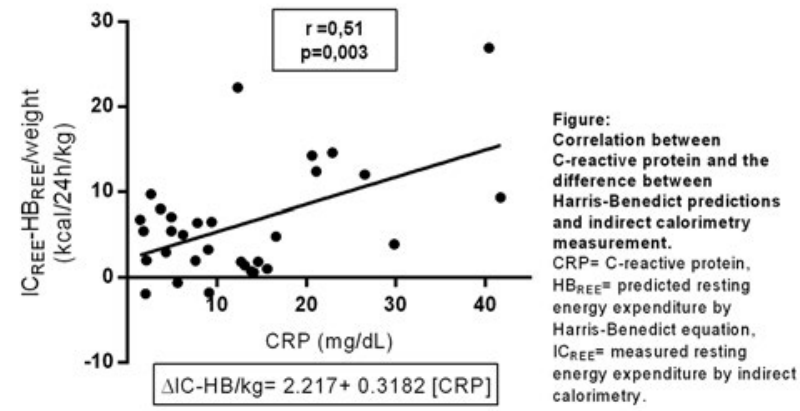


explained by the fact that this equation includes minute volume ventilation as a variable. This factor can greatly change over time. Inflammatory state defined by C-reactive protein seems, not surprisingly, to influence resting energy expenditure. This is the first study in critically ill demonstrating clearly a significant correlation between any increase in C-reactive protein and resting energy expenditure, particularly in patients with sepsis.

Conclusion: In critically ill with sepsis, resting energy expenditure determined by indirect calorimetry is well correlated with C-reactive protein. This marker could serve as a correction factor when indirect calorimetry is not available.

\section{FC044}

\section{Inflammation in cancer and immunonutrition:} is the neutrophil: lymphocyte ratio

\section{a promising monitoring?}

G. Echeverria ${ }^{1}$, S. Giglioli ${ }^{1}$, P. Forget ${ }^{1}$, A. Steyaert ${ }^{1}$, B. Bertrand ${ }^{2}$,

M. de Kock ${ }^{1}$

${ }^{1}$ Anesthésie-réanimation, cliniques universitaires Saint-Luc,

Bruxelles, Belgique

${ }^{2}$ Nutrition clinique, cliniques universitaires Saint-Luc, Bruxelles,

Belgique

Introduction: In patients undergoing major surgery, pre-existing malnutrition and the patient's stress response to surgical trauma are amongst the most important factors affecting outcome. Our understanding of the inflammatory pathophysiology is still incomplete. We currently have no tool to identify patient subgroups who would best benefit from immunonutrition. In this study, we analyzed the preoperative variations of the Neutrophil: Lymphocyte Ratio (NLR), one of the most sensitive inflammatory markers, in cancer patients.

Patients et méthodes: With the agreement of our ethics committee, we reviewed the data of 116 patients scheduled for major abdominal cancer surgery. Blood samples were taken at the diagnosis, 4 to 8 weeks before surgery (T0), the week before surgery (T1) and, when given, after immunonutrition administration (T2). Immunonutrion consisted typically in ORAL IMPACT (Nestle Nutrition) witch

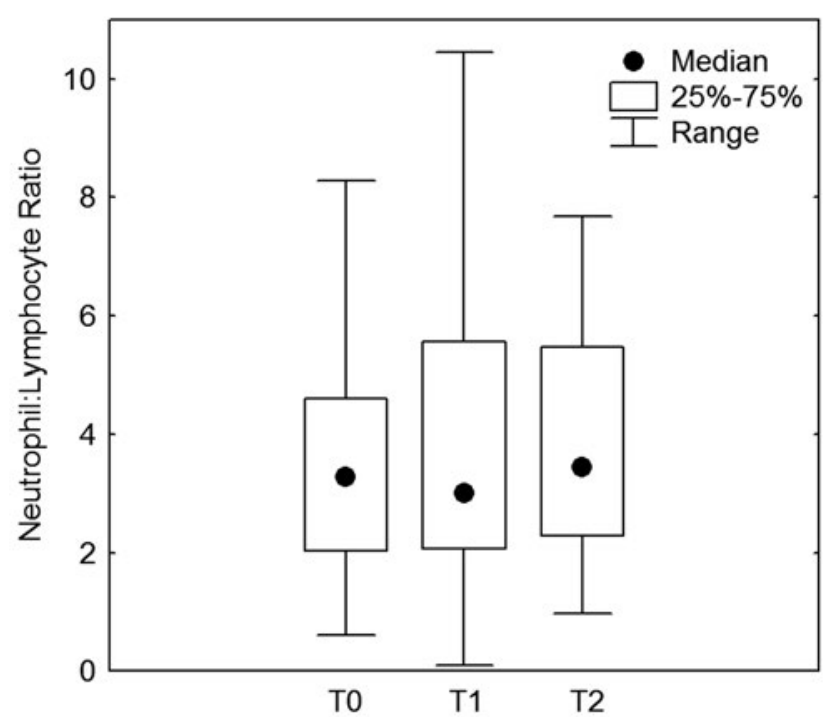

Figure Neutrophil: Lymphocyte ratio (NLR) in 116 patients 4-8 weeks before immunonutrition (T0), the week before surgery (T1) and after immunonutrition (T2). contains arginine, $\omega-3$ polyunsatured long chain fatty acids (PUFAs), RNA and antioxydants as selenium. The patient received 2 sachets/day for 5-7 days before surgery (depending on the patient's nutritional status). We calculated the NLR at each time. Wilcoxon matched pairs tests were used to compare measures at different time. $\mathrm{P}<0.05$ was considered significant.

Résultats: NLR did not change significantly during the study period, including after the immunonutrition period for the patients receiving it (T0: mean $\pm \mathrm{SD}$, median: [IQ25-75], $4.29 \pm 4.55,3.28$ [2.03-4.60]; $\mathrm{T} 1: 4.86 \pm 5.26 ; 3.0$ [2.05-5.58]; T2: $4.10 \pm 2.60,3.44$ [2.20-5.51]) $(\mathrm{P}>0.05)$ (Figure). Similarly, C-Reactive Protein did not change significantly (data not shown).

Conclusion: Although inflammation is implicated in the pathophysiology of cancer, preoperative variations of the NLR value were not significant in this series. Despite a presumed anti-inflammatory modulation and influence on immune parameters, immunonutrition was not associated with a significant variation of the NLR value.

\section{FC045}

\section{Conséquences d'un déficit énergétique acquis durant la première semaine d'hospitalisation en réanimation polyvalente}

F. Hervé ${ }^{1}$, J.-L. Frances ${ }^{2}$, G. Halley ${ }^{2}$, M. Moriconi ${ }^{2}$, A. Tonnelier ${ }^{2}$ ${ }^{1}$ Réanimation polyvalente, hôpital, Quimper, France

${ }^{2}$ Service de réanimation médico-chirurgicale, $\mathrm{CH}$ de Quimper, Quimper, France

Introduction : Des études antérieures ont déjà souligné l'association entre déficit calorique cumulé et morbi-mortalité. Sans surprise, ce déficit est plus important au cours de la première semaine de réanimation. Toutefois corrélation ne signifie pas causalité. En effet, plus les patients sont graves plus ils ont tendance à rester en réanimation et à développer des complications. Le but de ce travail est d'étudier les conséquences du déficit calorique constitué au cours de la première semaine d'hospitalisation en réanimation polyvalente sur le devenir des patients.

Patients et méthodes : Il s'agit d'une étude monocentrique rétrospective menée entre janvier et juin 2011 dans un service de réanimation polyvalente de 15 lits, dont 3 de surveillance continue. Les critères d'inclusion étaient : âge supérieur à 18 ans, admission en secteur de réanimation pendant au moins une semaine, alimentation artificielle durant au moins 2 jours. Les besoins caloriques théoriques ont été estimés par la formule d'Harris et Benedict ajustée par un facteur multiplicatif de stress. L'apport calorique hebdomadaire comprenait les perfusions glucosés ainsi que l'alimentation entérale et parentérale. Des corrélations entre la balance énergétique (BE) (apports délivrés - apports théoriques) et les données suivantes ont été recherchées : mortalité (réanimation et $\mathbf{J} 28$ ), durée de séjour (en réanimation et à l'hôpital), nombre d'infections nosocomiales, de jours sédatés, ventilés, dialysés et sous amines rapportés à la durée de séjour en réanimation, le score SOFA de J7. Les analyses statistiques univariées ont été effectuées par un test de Mann-Whitney (mortalités) et une corrélation de Spearman. Un résultat a été considéré comme significatif si $\mathrm{p}<0,05$.

Résultats : Trente patients ont été inclus dans l'étude dont $27 \%$ chirurgicaux. La moyenne d'âge est de 62 ans avec un IGS II moyen de 48. La mortalité en réanimation et à $\mathrm{J} 28$ est de $27 \%$. La durée moyenne de séjour en réanimation est de 33 jours et à l'hôpital de 60 jours. La moyenne du score SOFA à $\mathrm{J} 7$ est de 6 . La ventilation mécanique, les amines, l'épuration extra-rénale (EER) et la sédation représentent respectivement $78 \%, 28 \%, 9 \%$ et $45 \%$ de la durée de séjour en 
réanimation. Le nombre d'infections nosocomiales rapporté à la durée de séjour en réanimation est de 0,02 . L'objectif calorique moyen est de $30 \mathrm{kcal} / \mathrm{kg} / \mathrm{j}$. Sur le séjour de réanimation l'apport calorique moyen délivré est de $25 \mathrm{kcal} / \mathrm{kg} / \mathrm{j}$ soit $85 \%$ de l'apport énergétique théorique. Au cours de la première semaine d'hospitalisation l'apport énergétique moyen est de $17 \mathrm{kcal} / \mathrm{kg} / \mathrm{j}$ soit $57 \%$ de l'apport énergétique théorique. Ce déficit est constaté au cours des 6 premières semaines et n'est rattrapé ensuite que partiellement. Nous ne retrouvons pas de lien entre le déficit énergétique à $\mathrm{J} 7$ et les mortalités $(\mathrm{p}=0,47)$. Aucun autre lien n'est mis en évidence entre le déficit énergétique à $\mathrm{J} 7$ et l'incidence d'infections nosocomiales $(\mathrm{p}=0,76)$, les proportions du séjour sous ventilation mécanique $(p=0,18)$, dialyse $(p=0,29)$, amines $(p=0,79)$, sédation $(\mathrm{p}=0,7)$ et le score SOFA de $\mathrm{J} 7(\mathrm{p}=0,32)$.

Discussion : Le déficit énergétique constaté à J7 s'explique d'une part par une nutrition artificielle débutée en moyenne après 2,4 jours et d'autre part par une introduction progressive protocolisée de l'alimentation entérale. Malgré tout, cette balance énergétique négative de $-43 \%$ ne semble pas préjudiciable aux patients en terme de mortalités, de recours aux thérapeutiques de réanimation (sédation, amines, ventilation mécanique et EER), d'infections nosocomiales et de défaillances d'organes. Ce résultat conforte l'attitude d'une nutrition artificielle retardée de quelques jours une fois l'état clinique des patients stabilisés. L'association entre déficit calorique et morbi-mortalité déjà soulignée semble donc plus lié au déficit cumulé tout du long du séjour en réanimation.

Conclusion : Un déficit calorique de $43 \%$ constitué au cours de la première semaine d'hospitalisation en réanimation polyvalente ne semble pas préjudiciable aux patients.

\section{FC046}

\section{Impact du déficit énergétique sur les caractéristiques microbiologiques des bactériémies acquises} en réanimation chez les malades ventilés artificiellement K. Ekpe ${ }^{1}$, J.-L. Mainardi ${ }^{2}$, A. Novara-Flor ${ }^{3}$, C. Faisy ${ }^{3}$ ${ }^{1}$ Réanimation, institut Gustave-Roussy, Paris, France

${ }^{2}$ Service de microbiologie, hôpital européen Georges-Pompidou (HEGP), Paris, France

${ }^{3}$ Service de réanimation médicale, hôpital européen Georges-Pompidou (HEGP), Paris, France

Introduction : La bactériémie est la troisième cause d'infection nosocomiale en réanimation et elle est grevée d'une morbi-mortalité importante. Il existe une association entre déficit énergétique et complications infectieuses en réanimation, en particulier les bactériémies [1]. Par ailleurs, nous avons montré récemment que l'ampleur du déficit énergétique précocement constitué en réanimation était un facteur de risque indépendant de la survenue d'une pneumopathie acquise sous ventilation mécanique à Staphylocoque doré. Notre objectif était d'estimer l'impact du déficit énergétique sur les caractéristiques microbiologiques des bactériémies acquises chez les malades intubés d'emblée pour une pathologie aiguë.

Patients et méthodes : Nous avons mené une étude observationnelle rétrospective chez des patients ayant développé un premier épisode de bactériémie acquise (au moins $48 \mathrm{~h}$ après l'admission) au cours de leur période de ventilation mécanique invasive entre Janvier 2009 et Décembre 2012. Le bilan énergétique des patients était calculé chaque jour à partir des données des feuilles de prescription et des pancartes de soins et de surveillance. La dépense énergétique était calculée à l'aide de la méthode prédictive développée par Faisy en 2003 [2]. Les données microbiologiques fournies par les hémocultures (Gram, espèce, caractéristiques de multi-résistance) et les facteurs de risque habituellement décrits dans la littérature ont été également collectés. De même, les facteurs favorisant la sous-prescription de calories ou les situations ralentissant l'administration des calories prescrites étaient recherchés.

Résultats : Quatre vingt quatre patients ventilés plus de 72 heures consécutives ont été inclus dans l'analyse. Le déficit énergétique moyen était de $-1005 \pm 409 \mathrm{kcal} /$ jour de ventilation invasive. Seuls les patients ayant développé une bactériémie à Staphylocoque doré résistant à la méthicilline (SARM, $n=6$ ) avaient un déficit énergétique significativement plus important $(p=0,02)$ que les malades ayant une hémoculture à un autre germe $(\mathrm{n}=78)$. Cette différence n'était pas due à une dépense énergétique accrue chez les patients infectés à SARM. Les malades infectés à SARM ne différaient pas des autres patients pour leur motif d'admission, les facteurs de risque d'infections ou les caractéristiques de leur séjour en réanimation. Seules la prescription de calories $(p=0,001)$ et l'administration de ces calories $(p=0,002)$ étaient significativement diminuées chez les patients infectés à SARM. Les patients avec une bactériémie à Staphylocoque doré sensible ou non à la méthicilline $(\mathrm{n}=18)$ ou à Staphylocoque coagulase négative $(n=9)$ avaient aussi un déficit énergétique plus important mais la différence avec les patients ayant développé une bactériémie à un autre germe demeurait statistiquement non significative.

Conclusion : Le déficit en énergie précocement constitué en réanimation favorise les bactériémies à SARM chez les malades ventilés d'emblée pour une pathologie aiguë. Ces résultats complètent les données connues pour les PAVM et mettent en évidence le lien entre déficit énergétique et caractère de résistance aux antibiotiques du Staphylocoque doré.

1. Rubinson L, et al. (2004) Low caloric intake is associated with nosocomial bloodstream infections in patients in the medical intensive care unit. Crit Care Med 32:350-357

2. Faisy C, et al. (2011) Early ICU energy deficit is a risk factor for staphylococcus aureus ventilator-associated pneumonia. Chest 140:1254-1260

\section{FC047}

\section{La perte de masse musculaire évaluée par tomodensitométrie (TDM) abdominale avec coupe centrée sur la troisième vertèbre lombaire (L3) à l'admission en réanimation est un marqueur prédictif de mortalité}

A. Ait Hssain ${ }^{1}$, S. Dadet ${ }^{2}$, B. Pereira ${ }^{3}$, B. Souweine ${ }^{1}$, S. Antoun ${ }^{4}$, E. Dumousset ${ }^{5}$, N. $\mathrm{Cano}^{6}$, R. Thibault ${ }^{7}$

${ }^{1}$ Service de réanimation polyvalente, CHU Gabriel-Montpied, Clermont-Ferrand, France

${ }^{2}$ Service de nutrition clinique, CHU Gabriel-Montpied, Clermont Ferrand, France

${ }^{3}$ Direction de recherche clinique et innovations,

CHU Clermont-Ferrand, Clermont-Ferrand, France

${ }^{4}$ Service des urgences, institut Gustave-Roussy, Villejuif, France

${ }^{5}$ Service de radiologie, CHU Gabriel-Montpied, Clermont-Ferrand, France

${ }^{6} U N H$, service de nutrition clinique, CRNH, CHU Gabriel-Montpied, Clermont-Ferrand, France

${ }^{7}$ Unité de nutrition, hôpitaux universitaires de Genève, Genève, Suisse

Introduction : La perte de masse musculaire s'associe à un moins bon pronostic. Peu de données existent en réanimation. Les objectifs étaient de déterminer à l'admission i) la faisabilité de la mesure de la 
masse musculaire par TDM centré sur L3 (L3-TDM), ii) l'impact du niveau de masse musculaire sur la mortalité la durée de séjour, la durée de ventilation méanique, et les bactériémies nosocomiales (BN), et iii) la sensibilité et la spéificitéde l'indice de masse corporelle (IMC) pour estimer la masse musculaire mesuré par L3-TDM.

Patients et méthodes : Parmi les 200 patients ayant eu une TDM abdominale sur une période de 3 ans, nous avons sélectionné les 129 patients avec durée de séjour $\geq 48 \mathrm{~h}$ et TDM dans les $72 \mathrm{~h}$ postadmission. L'index de masse musculaire squelettique (IMMS) a été déterminé à partir de la surface musculaire squelettique par un seul opéateur sur une coupe TDM centré sur L3 à l'aide du logiciel Slice-O-matic ${ }^{\circledR}$ selon la formule : IMMS = surface musculaire squelettique L3 (cm2)/taille (m) 2. La masse musculaire était abaissé $\mathrm{si}<38,5 \mathrm{~cm} 2 / \mathrm{m} 2$ (femmes) et $<52,4 \mathrm{~cm}^{2} \mathrm{~m}^{2}$ (hommes) (Prado, Lancet Oncol 2008). Les variables ont été comparés (tests de Chi-2 ou Kuskall-Wallis). La sensibilité, la spécificité et le cut-off d'IMC ou d'IMMS associé à la diminution de masse musculaire ou à la mortalité respectivement, ont été déterminé par courbes ROC.

Résultats : L'IMMS a pu être calculé chez $123(95 \%)$ patients $(49 \mathrm{H}$, $65 \pm 14$ ans, IGS : $62 \pm 22$ ). L'IMMS médian était $49,7 \pm 11 \mathrm{~cm} 2 / \mathrm{m} 2$ (hommes) et 47,7 $\pm 13,8 \mathrm{~cm} 2 / \mathrm{m} 2$ (femmes) $(\mathrm{p}=0,39)$. Chez les hommes, l'IMMS à l'admission était plus élevé chez les survivants que les décédés : $53,4 \pm 10,6$ vs $46,1 \pm 10,3 \mathrm{~cm} 2 / \mathrm{m} 2, \mathrm{P}=0,004$. La sensibilité et la spécificité d'un IMMS $<54,0$ pour prédire la mortalité étaient de $50 \%$ et de $86 \%$, respectivement (aire sous la courbe (AUC) 0,70 $[0,57-0,82])$. Population totale :

\begin{tabular}{|c|c|c|c|c|c|c|}
\hline & $\begin{array}{l}\text { Sex ratio } \\
(\mathrm{H} / \mathrm{F})\end{array}$ & $\begin{array}{l}\text { IMC } \\
\text { Moy } \pm \text { DS }\end{array}$ & $\begin{array}{l}\text { Mortalité Réanimation/ } \\
\text { hôpital- n(\%) }\end{array}$ & $\begin{array}{l}\text { Durée séjour Réanimation/ } \\
\text { hôpital médiane (min-max) }\end{array}$ & $\begin{array}{l}\text { Durée ventilation } \\
\text { mécanique moy } \pm \text { DS }\end{array}$ & $\mathrm{BN}-\mathrm{n}(\%)$ \\
\hline \multirow{2}{*}{$\begin{array}{l}\text { Masse musculaire } \\
\text { normale }(\mathrm{n}=66)\end{array}$} & \multirow[t]{2}{*}{$27 / 39$} & \multirow[t]{2}{*}{$30,4 \pm 6,8$} & $25(38)$ & $11(6-19)$ & $14,2 \pm 18,0$ & \multirow[t]{2}{*}{$3(5)$} \\
\hline & & & $32(48)$ & $21(12-36)$ & $21(12-36)$ & \\
\hline \multirow{2}{*}{$\begin{array}{l}\text { Masse musculaire } \\
\text { abaissée }(\mathrm{n}=57)\end{array}$} & \multirow[t]{2}{*}{$45 / 12$} & \multirow[t]{2}{*}{$24,9 \pm 4,4$} & $26(46)$ & $12(5-20)$ & $18,0 \pm 18,5$ & \multirow[t]{2}{*}{$5(9)$} \\
\hline & & & $33(58)$ & $20(15-40)$ & $20(15-40)$ & \\
\hline$P$ & $<0,001$ & $<0,001$ & $0,39 / 0,30$ & $0,60 / 0,70$ & 0,33 & 0,47 \\
\hline
\end{tabular}

La sensibilité et la spécificité d'un IMC $<22,0$ (femmes) et $<24,9$ (hommes) étaient de, respectivement, $92 \%$ et $42 \%$ (AUC 0,76 $[0,62-, 90])$, et $96 \%$ et $42 \%$ (AUC $0,77[0,66-0,88]$ ) pour prédire la perte musculaire.
Conclusion : La mesure de la masse musculaire par L3-TDM est applicable à l'admission en réanimation à partir de TDM standards à visée diagnostique, et pourrait prédire la mortalité chez les hommes. Un IMC $<22,0$ (femmes) et $<24,9$ pour les hommes sont sensibles pour diagnostiquer la perte musculaire. 